\title{
Aquaporin MRI
}

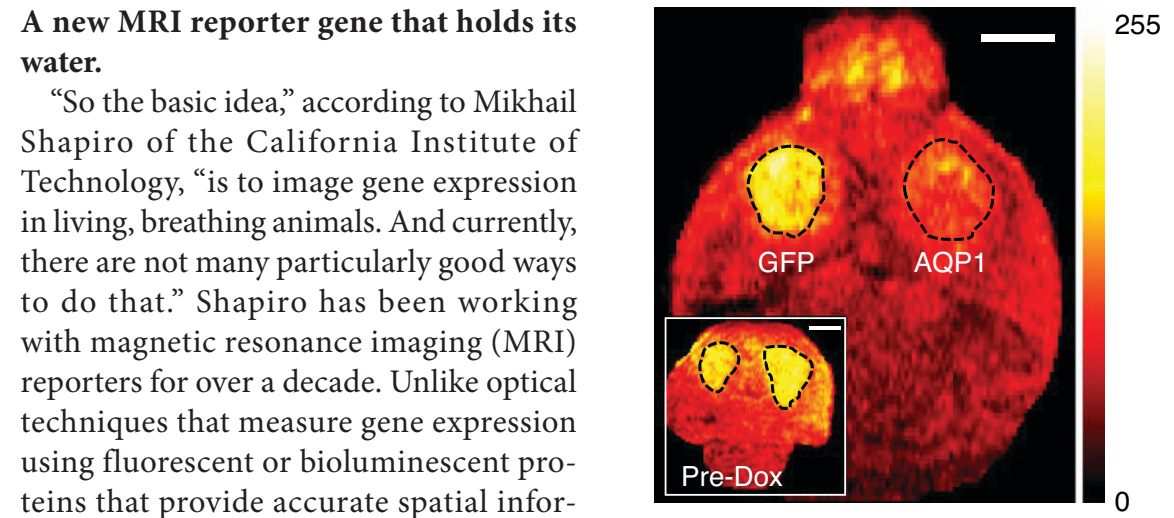

teins that provide accurate spatial information only a millimeter or so into tissue, MRI is highly penetrant and can resolve images deep inside an organism. Finding the right reporter to use with MRI imaging of gene expression, has long been a goal of researchers.

Previous research has frequently relied on metal-based reporters, which need metals to be available in tissues "at the right quantity, and at the right time, and with the right chemistry to work efficiently," explains postdoctoral scholar Arnab Mukherjee, a somewhat complicated process with an inherent risk of metal toxicity. And other candidates, derived from bacteria, carry potential immunogenicity issues. Mukherjee comments, "What we wanted to do was to use an autologous, human-based gene that obviates the need to use metals for MRI contrast." A common imaging method, diffusion-weighted MRI, essentially measures the movement of water, so why not look there?

For their reporter, the team turned to a group of highly conserved channel proteins found from bacteria to people known as aquaporins (Nat. Commun. 7, 13891; 2016). Shapiro refers to aquaporins as "professional water-conducting channels," selectively letting water in and out of a cell without changing any other chemical balances. They hypothesized that the water diffusing across cell membranes expressing
Tumors expressing APQ1 appear darker in diffusion-weighted MRI images than GFPexpressing controls and pre-doxycycline activation. Adapted from Nat. Commun. 7, 13891; 2016.

aquaporin would be picked up using diffusion-weighted MRI; specifically with this method, cells that express aquaporin will appear darker in the resulting images relative to surrounding cells.

Having identified an aquaporin with fast water conducting capacity, called human aquaporin 1 (AQP1), they tested its ability to image mammalian cells, starting with tumors. Tumors often have low expression of aquaporin, making them a good starting place to provide proof-of-concept. The team created several distinct cell lines that express AQP1 and compared diffusion-weighted images of those cells to controls tagged with a green fluorescent protein (GFP). The purpose of GFP was not for fluorescence, as it is typically used, but to control for reporter gene expression. Cells with either reporter were visible in diffusion MRI images, but AQP1-expressing cells were much darker, reflecting the increased water diffusion in those lines. They also confirmed that expression of AQP1 did not cause toxicity or other changes in cell morphology.

Encouraged by their in vitro results, they looked to see how AQP1 would function in an animal model. For these experiments,
$\mathrm{CHO}$ cells engineered to express AQP1 under a doxycycline-inducible promoter were implanted into the left hemisphere of female immunocompromised NOD/SCID mice; the right hemisphere received control cells expressing GFP. Leaving the tumors to grow for about a week, the team then imaged the mice before and after AQP1 expression was turned on with a dose of doxycycline. Before, both tumors looked about the same-essentially two small blobs of light in the brain. After a shot of doxycycline, the AQP1-expressing tumor again darkened as expected.

Next up for future work is optimization. AQP1 allowed detection of relatively low levels of protein expression, about 500 nanomolar, but, says Shapiro, "We'd like to push the sensitivity, with a combination of engineering aquaporins to increase water conduction and engineering how we do the MRI to make it as sensitive as possible." Increasing the sensitivity, he explains, will permit detection at even lower levels of expression, as well as when the cells in question are part of a mixed population. These refinements should make the technique relevant in a range of scenarios for any group with access to standard MRI equipment.

In addition to tracking tumors, Shapiro and Mukherjee think the aquaporin reporter may prove useful for cell and gene therapy research, giving researchers the ability to monitor the location, viability, and genetic functionality of a given therapy, as well as for studies of basic biology and development. Mukherjee summarizes, "It's a reporter gene that lets you study biology in the context of living, breathing animals. So if you really want to probe all these fundamental questions, or questions with an applied clinical angle but study them in the context of animal models, I think all of these would be great areas for applications of aquaporin as a non-invasive MRI reporter."

Ellen P. Neff 